\title{
Current Status of the Oak Ridge Monte Carlo Codes: MORSE/SAS4 and KENO
}

Margaret B. Emmett and D. F. Hollenbach

Oak Ridge National Laboratory,

P. O. Box 2008,

Oak Ridge, TN 37831-6370

(865) 574-5276 office number

(865) 576-3513 fax number

emmettmb@ornl.gov

Submitted to the

Monte Carlo 2000 - Advanced Monte Carlo Radiation Physics, Particle Transport Simulation Applications,

October 23-26, 2000,

Lisbon, Portugal

The submitted manuscript has been authored by a contractor of the U.S. Government under contract No. DE-AC05-00OR22725. Accordingly, the U.S. Government retains a nonexclusive, royalty-free license to publish or reproduce the published form of this contribution, or allow others to do so, for U.S. Government purposes.

*Managed by UT-Battelle, LLC, under contract DE-AC05-00OR22725 with the U.S. Department of Energy. 


\title{
Current Status of the Oak Ridge Monte Carlo Codes: MORSE/SAS4 and KENO
}

\author{
Margaret B. Emmett ${ }^{1}$ \\ Daniel F. Hollenbach ${ }^{2}$ \\ ${ }^{1}$ Oak Ridge National Laboratory, MS-6370, Oak Ridge, TN 37831-6370, \\ Phone (865/574-5276), Fax (865/576-3513), email emmettmb@ ornl.gov \\ 2 Oak Ridge National Laboratory, MS-6370, Oak Ridge, TN 37831-6370, \\ Phone (865/576-5258), Fax (865/576-3513), email hollenbachdf@ornl.gov
}

Topics of interest: neutron-gamma codes

\begin{abstract}
Over the years, the Oak Ridge National Laboratory staff have developed a number of Monte Carlo codes. KENO V.a and KENO-VI are criticality safety codes that can be run stand-alone or by using the criticality safety analysis sequences CSAS and CSAS6. MORSE is a shielding analysis code that can be run stand-alone or through use of the shielding analysis sequences SAS3 and SAS4. All of these codes are part of the SCALE code system, which is widely distributed and used all over the world. As with any major computer code system, changes and improvements are inevitable. These changes occur for multiple reasons, including release of new compilers, faster and improved computers, and changing customer requirements. This paper provides a current overview of these Monte Carlo codes and a look at possible future enhancements.
\end{abstract}

\section{Monte Carlo Criticality Safety Codes}

The first part of this presentation covers the criticality safety codes and code sequences that are available in the SCALE [1] code system.

\subsection{KENO V.a}

KENO V.a is a multigroup Monte Carlo criticality program used to calculate the k-effective of a three-dimensional (3-D) system. KENO V.a's geometry input allows the use of nonconcentric cuboidal, cylindrical, and spherical shapes and has "array-of-arrays" and "holes" capabilities. The array-of-arrays option allows construction of arrays from other arrays. The hole option allows placing a unit or an array at any desired location within a geometry region. This option is quite useful for describing shipping casks and reflectors that have gaps. KENO V.a also has the capability of supergrouping energy-dependent information such as cross sections. Supergrouping is a process that allows a problem to run with only part of the data in core, rather than the entire energy range. 


\subsection{KENO-VI}

KENO-VI contains all the features of KENO V.a plus a more flexible geometry package. The KENO-VI geometry package is capable of modeling any volume that can be constructed using quadratic equations. Other geometry features such as intersections, body rotations, hexagonal arrays, and array boundaries make the code more flexible. The ability to intersect volumes makes it possible to model such things as pipe intersections exactly, which were previously impossible with KENO V.a.

\subsection{The CSAS Sequences}

CSAS was developed to provide automated, problem-dependent cross-section processing followed by calculation of the neutron multiplication factor for the system being modeled. CSAS executes BONAMI, NITAWL-II, and KENO V.a. Control sequences ending in " $\mathrm{X}$ " also execute XSDRNPM to provide a cell-weighted mixture cross section; CSASI and CSASIX use ICE to provide a Monte Carlo formatted mixed cross-section library. CSAS6 is a version of CSAS written explicitly for KENO-VI.

\subsection{The KENO3D Visualization Tool}

A recent addition to the KENO code systems is KENO3D [2]. KENO3D is a state-of-the-art visualization tool for KENO geometry models that allows the user to interactively display a 3-D geometry model. The KENO V.a version is currently available to the public; the KENO-VI version should be available sometime during the fall of FY 2000. A paper on KENO3D is being presented at this conference.

\section{Monte Carlo Shielding Codes}

This section will present the Monte Carlo shielding codes that are modules in the SCALE code system.

\subsection{MORSE}

The Multigroup Oak Ridge Stochastic Experiment code, MORSE [3], is a 3-D multipurpose neutron and gamma-ray transport code that has been in use for nearly 30 years and has undergone several evolutions during that time. Oak Ridge National Laboratory (ORNL) has two versions that are distributed by the Radiation Safety Information Computational Center (RSICC): MORSE-CGA and MORSESGC. MORSE-SGC is part of the SCALE [1] computer code system, both as a stand-alone program and as part of two of the shielding analysis sequences, SAS3 and SAS4. 
Both SGC and CGA use the Multiple Array System (MARS) geometry package. The primary difference in the two versions is the cross-section structure. MORSE-SGC has a supergrouping capability that allows a problem to run with only part of the cross-section groups in core rather than the entire energy range. Because of the amount of memory available in today's computers, this feature is not nearly as important now as it was when the code was originally developed. The other major difference in the two versions is that SGC has built-in routines for the source selection and estimation routines, whereas the user can supply his or her own routines and override the provided versions in CGA.

\subsection{SAS4}

SAS4 was developed to solve spent fuel shipping cask problems and, as a result, has an automated geometry-modeling procedure. When the SAS4 shielding analysis sequence was being developed, one of the objectives was to automate the selection of biasing parameters for MORSE and the preparation of cross-section data. As a result of this, SAS4 runs cross-section resonance-processing modules BONAMI and NITAWL and an adjoint case using the one-dimensional code XSDRNPM prior to executing the MORSE module. The adjoint XSDRNPM results are used to generate biasing parameters for MORSE. Another feature of SAS4 is that the PICTURE program, which generates 2-D color plots, or printer plots can be run from within SAS4. A number of changes were required for MORSE-SGC to implement a new SAS4 option for surface detectors and subdetectors. Prior to these changes, SAS4 restricted the number of surface detectors to four preset locations for all of the geometry options. This update allows the user to specify up to 36 surface detectors when using the MARS geometry option rather than the simplified geometry input options; furthermore, each surface can be subdivided into spatial and azimuthal segments. This option is only available when running SAS4; i.e., it is not an option when running MORSE-SGC stand-alone.

\subsection{SAS3}

The SAS3 shielding analysis sequence runs the cross-section modules and MORSE. At present, there is no automated selection of biasing parameters. SAS3 can, however, solve many problems that the specialized SAS4 can not handle. Examples of such problems are streaming through ventilation ducts and cask penetrations and applications to more generalized shielding problems. Currently, SAS3 or MORSE-SGC can treat any of these applications. However, the user must generate and input very detailed biasing parameters.

\section{Graphical User Interfaces}

One current project under development is a SCALE shielding analysis graphical user interface (GUI) for Windows 95/98/NT/2000. This GUI, known as ESPN for 
Easy Shielding Processor iNput, will provide an easy-to-use system to assist the occasional and inexperienced SCALE users in the setup and execution of SAS4/MORSE. The material information cross-section portion is nearly identical to that in CSPAN (Criticality Safety Input Processor for Analysis), the GUI for the CSAS criticality sequence that is already complete. CSPAN and ESPN are specifically designed for SCALE 4.4 or 4.4 a on a WINDOWS PC. For ESPN, the primary development effort will be in the geometry input specifications. A preliminary version of ESPN, without the geometry input, is being tested at ORNL. CSPAN is being distributed with the SCALE 4.4a package. Both the initial release and future updates to ESPN and CSPAN can be found on the SCALE Download page (http://www.cped.ornl.gov/scale/download.html).

\section{SCALE 5 Development}

Work currently in progress on the SCALE code system includes the development of SCALE 5. Although version 4.4a, the current release of SCALE, is FORTRAN90 compatible, it requires using several of the extensions to the language. The FORTRAN routines for version 5 will be pure FORTRAN-90 and, as such, will not require use of extensions. SCALE 5 will, however, still have some library routines written in the $\mathrm{C}$ language. Several new features are being added to the Monte Carlo codes.

\subsection{New Features of the Criticality Safety Codes}

Recent development for the SCALE 5 version of the KENO control sequences includes a new pointwise discrete-ordinates neutron-transport code called CENTRM (Continuous ENergy TRansport Model)[4] which is based on the 1-D discrete ordinates code XSDRNPM. One of the features of CENTRM is the ability to explicitly model flux distributions radially across a pin, an option that allows the user to model fuels and dissolvers in solutions containing fissile materials (e.g., a fuel pellet). CENTRM uses a 1-D point flux distribution to collapse the point cross sections read from a CENTRM point cross-section file. This method produces more accurate group cross sections. Enhancements, expected to be ready this fall, include the extension of the pointwise solution into the thermal energy range. A complete ENDF/B-V CENTRM library is available for use with ENDF/B-V group cross-section libraries. Presently, an ENDF/B-VI CENTRM library is available, but it contains no thermal data. An ENDF/B-VI multigroup cross-section library is much larger than an ENDF/B-V library because it contains more resonance data and more nuclides.

SCALE 5 criticality control sequences will also include NITAWL-III, which contains a more sophisticated method for the resonance parameter processing. NITAWL-III is the latest variation of the modules that have served as the primary resonance self-shielding module in SCALE. The primary functional difference between NITAWL-III and its predecessor, NITAWL-II, is the capability of using multipole parameters for the resolved resonance in addition to the Briet-Wigner 
parameters. The multipole parameter method can be used to generate cross-section values that are equivalent to the widely used Reich-Moore data.

KENO-VI for SCALE 5 has recently been updated to include the capability to calculate volumes. SCALE 5 will have the ability to explicitly enter multiple unit cells. Currently, only one unit cell is allowed; all other material is treated as infinite homogeneous media. Dancoff factors can be incorporated in the non-unit cell materials (thus treating them as part of a unit cell) by adding them in the MORE DATA section of the problem. The ability to have multiple unit cells will allow search cases to properly modify the cross sections of any material in a unit cell during a pitch search.

\subsection{Future Directions for the Monte Carlo Codes}

Because unbiased Monte Carlo methods are not practical for real reactor and away-from-reactor applications which are typically large and geometrically complex and which also involve significant attenuation, the development of a 3-D Monte Carlo shielding analysis sequence with automatic variance reduction is highly desirable. As indicated above, the SAS4 sequence uses the 1-D code XSDRNPM to produce biasing parameters for MORSE; however, for generalpurpose 3-D shielding calculations, use of 3-D adjoint functions is necessary. For this reason, ORNL expects to develop a code sequence that uses the $S_{N}$ adjoint output from the 3-D discrete ordinates transport code TORT [5] to produce variance reduction parameters for use in MORSE. Development of this capability will require significant changes to MORSE and to the shielding sequence in which it is implemented. This sequence may be an update to the SAS3 sequence discussed above or it may be an additional shielding sequence. Sources of funding are being investigated; several proposals are in process. Additional biasing options for SAS3 and SAS4 are also possible, and future development projects are under consideration.

Development of additional features for the criticality safety codes will continue. In FY 2001, work is scheduled to begin on a continuous energy version of KENO V.a. As stated above, KENO3D development for KENO-VI is another ongoing project.

\section{Summary}

All of the codes discussed here run on multiple computer platforms, including DEC-Alpha, IBM RISC 6000 series, Sun and HP workstations, and on personal computers. Codes in the SCALE system are under configuration control and have been validated and verified. A set of shielding benchmarks has been documented in Ref. 6, and a set of criticality safety benchmarks has been documented in Ref. 7. Both sets are available on the SCALE website (http://www.cped.ornl.gov/scale). A set of neutronic benchmarks for mixed oxide fuel has been calculated using the SCALE/CENTRM sequence and is documented in Ref. 8. 


\section{References}

1. SCALE: A Modular Code System for Performing Standardized Computer Analyses for Licensing Evaluation, NUREG/CR-0200, Rev. 5 (ORNL/NUREG/CSD-2/R5), Vols. I, II, and III, March 1997. Available from Radiation Safety Information Computational Center at Oak Ridge National Laboratory as CCC-545.

2. J. E. Horwedel and S. M. Bowman, KENO3D, A Visualization Tool for KENO V.a and KENO-VI Geometry Models, NUREG/CR-6662 (ORNL/TM1999/284), U.S. Nuclear Regulatory Commission, Oak Ridge National Laboratory, 2000.

3. M. B. Emmett, MORSE-CGA: A Monte Carlo Radiation Transport Code with Array Geometry Capability, ORNL-6174, Martin Marietta Energy Systems, Inc., Oak Ridge National Laboratory, April 1985.

4. M. Williams and M. Asgari, "Computation of Continuous-Energy Neutron Spectra with Discrete Ordinates Transport Theory," Nucl. Sci. Eng. 121, 173201 (1995).

5. W. A. Rhoades and D. B. Simpson, The TORT Three-Dimensional Discrete Ordinates Neutron/Photon Transport Code (TORT Version 3), ORNL/TM13221, Lockheed Martin Energy Research, Inc., October 1997.

6. B. L. Broadhead, M. B. Emmett, and J. S. Tang, Guide to Verification and Validation of the SCALE-4 Radiation Shielding Software, NUREG/CR-6484 (ORNL/TM-13277), U.S. Nuclear Regulatory Commission, Oak Ridge National Laboratory, December 1996.

7. M. B. Emmett and W. C. Jordan, Guide to Verification and Validation of the SCALE-4 Criticality Safety Software, NUREG/CR-6483 (ORNL/TM-12834), U.S. Nuclear Regulatory Commission, Oak Ridge National Laboratory, December 1996.

8. D. F. Hollenbach and P.B. Fox, Neutronics Benchmarks of Mixed Oxide Fuels Using the SCALE/CENTRM Sequence, ORNL/TM-1999/299, Lockheed Martin Energy Research Corp., Oak Ridge National Laboratory, February 2000 . 